\title{
Magnetic moments of $K$ isomers as indicators of octupole collectivity
}

\author{
N. Minkov ${ }^{1,2}$ and P. M. Walker ${ }^{2,3}$ \\ ${ }^{1}$ Institute of Nuclear Research and Nuclear Energy, Bulgarian Academy of Sciences, \\ Tzarigrad Road 72, BG-1784 Sofia, Bulgaria \\ ${ }^{2}$ Department of Physics, University of Surrey, Guildford GU2 7XH, UK \\ ${ }^{3}$ CERN, CH-1211 Geneva 23, Switzerland
}

\begin{abstract}
The relation between the quadrupole-octupole deformation and the structure of high- $K$ isomers in heavy even-even nuclei is studied through a reflection asymmetric deformed shell model including a BCS procedure with constant pairing interaction. Two-quasiparticle states with $K^{\pi}=4^{-}, 5^{-}, 6^{-}, 6^{+}$and $7^{-}$are considered in the region of actinide nuclei ( $\mathrm{U}, \mathrm{Pu}$ and $\mathrm{Cm}$ ) and rare-earth nuclei (Nd, Sm and $\mathrm{Gd}$ ). The behaviour of two-quasiparticle energies and magnetic dipole moments of these configurations is examined over a wide range in the plane of quadrupole and octupole deformations $\left(\beta_{2}\right.$ and $\left.\beta_{3}\right)$. In all considered actinide nuclei, the calculations show that there is pronounced sensitivity of the magnetic moments to the octupole deformation. In the rare-earth nuclei, the calculations for ${ }^{154,156} \mathrm{Gd}$ show stronger sensitivity of the magnetic moment to the octupole deformation than in the other considered cases.
\end{abstract}




\section{Introduction}

Shape is a basic property of atomic nuclei. Most nuclei have non-spherical shapes, and a nuclear excited state can differ in shape compared to its respective ground state. It is therefore useful to consider how different observables reflect the different shape degrees of freedom. In the present work, we investigate a range of two-quasiparticle (2qp) states in quadrupole-deformed even-even nuclei, where there is also the possibility of octupole deformation. Such states may be isomeric on account of the $K$ quantum number [1], that is the projection of the angular momentum on the symmetry axis. The isomerism opens the possibility to make measurements of the magnetic dipole moment, for example by timedependent perturbed angular distributions of decay $\gamma$-rays [2], by low-temperature nuclear orientation [3], or by laser hyperfine spectroscopy [4]. While magnetic dipole moments primarily give information about the orbits of individual quasiparticles, these are themselves influenced by the shape of the potential in which they move. In the following, attention is focussed on high- $K$ states that are known to be isomeric.

Recently it was shown that the sensitivity of isomer excitation energies and magnetic dipole moments to non-zero octupole deformations can be examined through a reflection asymmetric deformed shell model (DSM) including a BCS procedure with constant pairing interaction [5]. Detailed calculations were performed for the $2 \mathrm{qp} K^{\pi}=8^{-}$, $\{\nu 7 / 2[624] \otimes \nu 9 / 2[734]\}$ state in ${ }^{244} \mathrm{Pu}$ as a function of the quadrupole and octupole deformation parameters $\beta_{2}$ and $\beta_{3}$. It was shown that the magnetic dipole moment of the $K^{\pi}=8^{-}$state of ${ }^{244} \mathrm{Pu}$ exhibits a strong sensitivity to the octupole deformation, while being only weakly dependent on the quadrupole deformation. Similar behaviour of the magnetic moments was indicated for the $K^{\pi}=6^{-},\{\nu 5 / 2[633] \otimes \nu 7 / 2[743]\}$ state in ${ }^{234} \mathrm{U}$ and for the $K^{\pi}=66^{+},\{\nu 5 / 2[622] \otimes \nu 7 / 2[624]\}$ state in ${ }^{244} \mathrm{Cm}$. The above approach was strongly motivated by the expectation that future experiments will be able to make appropriate measurements of the magnetic moments.

The purpose of the present work is to extend the DSM+BCS consideration of high- $K$ isomers in the actinide region, and also to examine other regions, such as the rare-earth nuclei, where octupole deformation might be expected [6]. Detailed calculations are now reported for the actinides ${ }^{236} \mathrm{U},{ }^{238} \mathrm{Pu}$ and ${ }^{244} \mathrm{Cm}$, and for the rare earth nuclei ${ }^{154} \mathrm{Nd},{ }^{156} \mathrm{Nd}$, ${ }^{160} \mathrm{Sm},{ }^{154} \mathrm{Gd}$ and ${ }^{156} \mathrm{Gd}$. While the emphasis of our work is on the sensitivity of the magnetic moments to the shape degrees of freedom, we also show the 2qp energy surfaces and their minima, and compare them with the results of ground-state energy-surface calculations that use the "macroscopic-microscopic" Strutinsky procedure.

The structure of the work is as follows. In section 2 the DSM+BCS approach is briefly presented. Numerical results and discussion are given in section 3. In section 4 concluding remarks are given. 


\section{Reflection asymmetric single-particle model with pair- ing interaction}

A single-particle (s.p.) deformed shell model, allowing reflection asymmetry [7, 8, 9], is applied to calculating the nuclear shell structure, in order to examine the possibility for the coupling of high- $K$ orbitals near the Fermi level. The particular realization of the model includes a Woods-Saxon potential with axial quadrupole and octupole deformations for which a numerical code is available [10]. The Hamiltonian of the model is

$$
H_{\mathrm{sp}}=T+V_{\mathrm{ws}}+V_{\text {s.o. }}+V_{\mathrm{c}}
$$

where

$$
V_{\mathrm{ws}}(\mathbf{r}, \hat{\beta})=V_{0}\left[1+\exp \left(\frac{\operatorname{dist}_{\Sigma}(\mathbf{r}, \hat{\beta})}{a}\right)\right]^{-1}
$$

is the Woods-Saxon potential with $\hat{\beta} \equiv\left(\beta_{2}, \beta_{3}, \beta_{4}, \beta_{5}, \beta_{6}\right)$ and $\operatorname{dist}_{\Sigma}(\mathbf{r}, \hat{\beta})$ being the distance between the point $\mathbf{r}$ and the nuclear surface represented by

$$
R(\theta, \hat{\beta})=c(\hat{\beta}) R_{0}\left[1+\sum_{\lambda=2,3, \ldots} \beta_{\lambda} Y_{\lambda 0}(\cos \theta)\right]
$$

Here $c(\hat{\beta})$ is a scaling factor to keep the volume fixed. $V_{\text {s.o. }}$ and $V_{\mathrm{c}}$ are the spin-orbit and Coulomb terms whose analytic form is given in [10].

The Hamiltonian (1) is diagonalized in the axially symmetric, deformed harmonic oscillator (ADHO) basis $\left|N n_{z} \Lambda \Omega\right\rangle$, with the s.p. wave function being obtained as the expansion

$$
\mathcal{F}_{\Omega}=\sum_{N n_{z} \Lambda} C_{N n_{z} \Lambda}^{\Omega}\left|N n_{z} \Lambda \Omega\right\rangle .
$$

In the case of non-zero octupole deformation the wave function (4) appears with mixed parity. Then the parity of a given s.p. orbital is characterized by the expectation (average) value of the parity operator

$$
\begin{aligned}
\left\langle\hat{\pi}_{\mathrm{sp}}\right\rangle & =\left\langle\mathcal{F}_{\Omega}\left|\hat{\pi}_{\mathrm{sp}}\right| \mathcal{F}_{\Omega}\right\rangle \\
& =\sum_{N n_{z} \Lambda}(-1)^{N}\left|C_{N n_{z} \Lambda}^{\Omega}\right|^{2} .
\end{aligned}
$$

In the present work we are restricted to the situation where the average parity remains close to the good values of +1 or -1 , i.e. where the parity is still an asymptotically good quantum number. More extended discussion of parity mixing and the construction of a total collective+intrinsic state with good parity is given in Ref. [11].

The pairing effect is taken into account through a BCS procedure with constant pairing interaction applied to the DSM s.p. levels. The pairing constants $G_{n / p}$ for neutrons(n)/protons(p) are taken as [12] (see page 311):

$$
G_{n / p}=\left(g_{0} \mp g_{1} \frac{N-Z}{A}\right) / A \text {. }
$$


In Ref. [12] it is suggested that $g_{0}=19.2 \mathrm{MeV}$ and $g_{1}=7.4 \mathrm{MeV}$. Here a slightly different value for the first parameter $g_{0}=17.8 \mathrm{MeV}$ is used. It was introduced in Ref. [5] to provide a better overall energy scale to examine the $K^{\pi}=8^{-}$isomeric state of ${ }^{244} \mathrm{Pu}$ and its dependence on quadrupole and octupole deformations. This value appears also to be reasonable in the present extended application of the formalism in the regions of actinide and rare-earth nuclei. The BCS procedure is applied, as suggested in Ref. [12], within energy windows including $(15 N)^{1 / 2}$ orbitals for neutrons and $(15 Z)^{1 / 2}$ orbitals for protons below and above the Fermi surface. As a starting point in the numerical solution of the gap equation, the phenomenological value $\Delta=12 \cdot A^{-1 / 2}$ is used for the pairing gap, and the average value between the energies of the last occupied orbital and the first unoccupied orbital is used for the chemical potential $\lambda$. It should be noted that in the nuclei under consideration, the values obtained for the neutron pairing gap $\Delta_{n}$ vary over a narrow range below and above $1 \mathrm{MeV}$, depending on the quadrupole and octupole deformations. Further, since the pairing gap plays an important role in the 2qp energy (see below), its dependence on the quadrupole-octupole deformations contributes to the eventual forming of isomeric energy minima. with

The energy of a $2 q p$ configuration with a broken pair is taken as $E_{2 q p}^{K \pi}=E_{1 q p}^{\Omega_{1} \pi_{1}}+E_{1 q p}^{\Omega_{2} \pi_{2}}$,

$$
E_{1 q p}^{\Omega \pi}=\sqrt{\left(E_{s p}^{\Omega \pi}-\lambda\right)^{2}+\Delta^{2}}
$$

being the one-quasiparticle energy. The $K$-value is determined as $K=\Omega_{1}+\Omega_{2}$, while the parity of the configuration is $\pi=\pi_{1} \cdot \pi_{2}$ ( or $\pi=\operatorname{sign}\left\langle\pi_{1}\right\rangle \cdot \operatorname{sign}\left\langle\pi_{2}\right\rangle$, in the case of non-zero octupole deformation).

The magnetic moment of the 2qp configuration is determined as [13]

$$
\mu=\mu_{N}\left[g_{R} \frac{I(I+1)-K^{2}}{I+1}+g_{K} \frac{K^{2}}{I+1}\right]
$$

with $\mu_{N}=e \hbar /(2 m c), g_{R}=Z / A$ and

$$
g_{K}=\frac{1}{K} \sum_{n=1,2}\left\langle\mathcal{F}_{\Omega_{n}}\left|g_{s} \cdot \Sigma+g_{l} \cdot \Lambda\right| \mathcal{F}_{\Omega_{n}}\right\rangle
$$

where $\Sigma=\Omega \mp \Lambda$ is the intrinsic spin projection, and $g_{l}$ and $g_{s}$ are the standard gyromagnetic ratios. The proton and neutron $g_{s}$ values are attenuated by a commonly used factor of 0.6 compared to the free values. As mentioned above, the present consideration is mainly restricted to the situations with only small parity mixing in the s.p. states. Typically the parity admixtures in the wave function do not exceed $10-15 \%$, which means that the contribution of the mixed components to the gyromagnetic ratio $g_{K}$, Eq. (9), is of second order and can be neglected at the current stage of the study. In this sense, the present situation corresponds to an approximate parity projection. In the general case of larger parity mixing, projection techniques can be applied as considered in Refs [6] and [11]. In the present work, larger parity mixing is found in certain regions of the quadrupole-octupole deformation space, and this is evident in the calculated 2qp energies and magnetic moments 
(see below). In these regions specific "leading" wavefunction components can disappear or be in two different s.p. states. These cases can be excluded from further consideration and do not affect the conclusions that are reached.

\section{Numerical results and discussion}

The present model approach has been applied to high- $K$ excited states in several even-even actinide and rare-earth nuclei where 2 qp isomers are known to exist. Specifically, detailed calculations have been performed for two-quasineutron configurations with $K^{\pi}=4^{-}$in ${ }^{236} \mathrm{U}$ [14], $K^{\pi}=4^{-}$in ${ }^{238} \mathrm{Pu}[15], K^{\pi}=6^{+}$in ${ }^{244} \mathrm{Cm}[16,17], K^{\pi}=4^{-}$in ${ }^{154} \mathrm{Nd}$ [18], $K^{\pi}=5^{-}$in ${ }^{156} \mathrm{Nd}[19], K^{\pi}=5^{-}$in ${ }^{160} \mathrm{Sm} \mathrm{[18],} K^{\pi}=7^{-}$in ${ }^{154} \mathrm{Gd}[20]$ and $K^{\pi}=7^{-}$in ${ }^{156} \mathrm{Gd}$ [21]. These are considered to be typical examples, with quasiparticle configurations that may occur also in other nuclides in the same mass regions. Furthermore, most of the 2qp configurations have anti-parallel intrinsic-spin couplings, since this is energetically favoured in even-even nuclei [22]. The sensitivity of the intrinsic-spin orientations to the deformation degrees of freedom then gives rise to the associated sensitivity of the magnetic dipole moments.

In the nuclei ${ }^{236} \mathrm{U}$ and ${ }^{238} \mathrm{Pu}$ the $K^{\pi}=4^{-}$state is considered to be formed by the configuration $\{\nu 1 / 2[631] \otimes \nu 7 / 2[743]\}[14,15]$. In both nuclei the calculations indicate pronounced minima in the 2qp energy in the $\left(\beta_{2}, \beta_{3}\right)$ plane. This is shown in Fig. 1 (upperleft plot) for ${ }^{236} \mathrm{U}$ and in Fig. 2 (upper-left plot) for ${ }^{238} \mathrm{Pu}$. In the $\beta_{2}$ direction the minima are found at $\beta_{2} \sim 0.235$, a little higher than the value $\beta_{2}=0.215$ obtained in the "macroscopicmicroscopic" approach of Möller et al. [23], and lower than the experimental values $\beta_{2}=$ 0.282 for ${ }^{236} \mathrm{U}$ and $\beta_{2}=0.286$ for ${ }^{238} \mathrm{Pu}$ [24]. In the $\beta_{3}$ direction the minima for both nuclei are found at non-zero octupole deformation with $\beta_{3}=0.07-0.08$. The key point for the present work is that, in both nuclei, the magnetic moment exhibits pronounced sensitivity to the octupole deformation and a weak dependence on the quadrupole deformation, as illustrated in the upper-right plots of Figs. 1 and 2. In the lower plots of the same figures the magnetic moment $\mu$ is shown as a function of $\beta_{3}$ for three different $\beta_{2}$ values corresponding to the "macroscopic-microscopic" quadrupole deformation, the deformation in the energy minimum, and the experimentally estimated deformation. In all cases, strong variation of $\mu$ with $\beta_{3}$ is observed. The octupole dependence of the neutron orbitals near the Fermi level of ${ }^{236} \mathrm{U}$ is illustrated in Fig. 3. From the left plot it is seen that for $\beta_{2}=0.235$ the orbitals $1 / 2[631]$ and $7 / 2[743]$ are very close to each other and cross at quite small octupole deformation, $\beta_{3} \sim 0.01-0.02$, and further diverge with increasing $\beta_{3}$. From the right plot it is seen that if the "macroscopic-microscopic" hexadecapole deformation $\beta_{4}=0.1$ [23] is added the crossing point of these two orbitals shifts to larger octupole deformation, $\beta_{3} \sim 0.08$. Based on this observation, one could suggest that the hexadecapole deformation is important in determining the 2qp energy minimum. However, the energy minima in Figs. 1 and 2 are obtained without considering $\beta_{4}$ in the calculation. Then one should remark that the quasiparticle energy (7) is determined not only by the s.p. energies, but also through the pairing gap which depends on the quadrupole-octupole deformation as mentioned in the previous section. Thus it appears that the formation of the $2 \mathrm{qp}$ energy minima involves a more complicated mechanism due to the interplay between the s.p. degrees of freedom 
and the pairing residual interaction.

In ${ }^{244} \mathrm{Cm}$ the $K^{\pi}=6^{+}$isomeric state is formed by the $\{\nu 5 / 2[622] \otimes \nu 7 / 2[624]\}$ configuration [16]. This nucleus was partly considered in Ref. [5], where the sensitivity of the magnetic moment of the $6^{+}$state to the octupole deformation was shown. In Fig. 4 (upperleft) the behaviour of the 2qp energy in the $\left(\beta_{2}, \beta_{3}\right)$ plane is shown. A clearly pronounced minimum around $\beta_{2}=0.25-0.26$ and $\beta_{3} \sim 0.1$ is observed. As in the previous nuclei, it is placed between the "macroscopic-microscopic" value $\beta_{2}=0.234$ [23], and the experimental value $\beta_{2}=0.297$ [24]. In Fig. 4 (upper-right) a two-dimensional plot for the magnetic moment $\mu$ is also given. Again, a strong change in $\mu$ is observed in the $\beta_{3} \neq 0$ direction, while for $\beta_{3}=0$ the dependence of $\mu$ on the quadrupole deformation $\beta_{2}$ is quite a weak. The sensitivity of the magnetic moment to the octupole deformation is illustrated in more detail in the lower plot of Fig. 4 where $\mu$ is shown as a function of $\beta_{3}$ at three different quadrupole deformations. The octupole dependence of the neutron orbitals near the Fermi level of ${ }^{244} \mathrm{Cm}$ is shown in Fig. 5 for $\beta_{2}=0.25$ (the 2qp energy minimum) and for $\beta_{2}=0.297$ (the experimental value). One sees that at both $\beta_{2}$ deformations the orbitals $5 / 2[622]$ and $7 / 2[624]$ evolve in parallel and close to each other with varying octupole deformation. Also it is seen that at the experimentally suggested $\beta_{2}=0.297$ both orbitals are separated by the orbital $1 / 2[501]$ up to $\beta_{3}=0.09$, which implies that the presently obtained minimum deformation, $\beta_{2}=0.25$, might be more favourable. However, as discussed above, the formation of the $2 \mathrm{qp}$ minimum is the result of a subtle interplay between the s.p. and pairing degrees of freedom.

We next consider the region of rare-earth nuclei. Here the expectation is that $2 q p$ energy minima could be observed at non-zero $\beta_{3}$ in the nuclei where the presence of octupole collectivity is known. Among the best representatives are the $N=90$ isotones ${ }^{150} \mathrm{Nd}$, ${ }^{152} \mathrm{Sm},{ }^{154} \mathrm{Gd}$ and ${ }^{156} \mathrm{Dy}[25]$. At present, only in one of these nuclei, ${ }^{154} \mathrm{Gd}$, is an isomeric state observed, with $K^{\pi}=7^{-}$(see below).

The nucleus ${ }^{154} \mathrm{Nd}$ is the closest isotope to ${ }^{150} \mathrm{Nd}$ for which an isomeric state is observed [18], having $K^{\pi}=4^{-}$with the $\{\nu 5 / 2[642] \otimes \nu 3 / 2[521]\}$ two-quasineutron configuration. The present result for the 2qp energy is shown in Fig. 6 (upper-left). An indication for a minimum is found in the region $\beta_{2}=0.28-0.3$ and $\beta_{3}=0.08-0.12$. It should be immediately noted that the calculations show a specific irregular behaviour of the orbitals forming the above configuration in certain deformation regions. In Fig. 6 (lower-left corner of upper-left plot) the DSM provides one more orbital with the same quantum numbers $3 / 2[521]$ as the leading s.p. wave-function component. This effect is due to the appearance of strong parity mixing as mentioned at the end of Section 2. In this situation the energy of the $\{\nu 5 / 2[642] \otimes \nu 3 / 2[521]\}$ configuration is poorly determined. Therefore this part of the $\left(\beta_{2}, \beta_{3}\right)$ plot should be excluded from consideration. Nevertheless, the remaining part of the plot still provides a clear indication for the 2qp energy minimum at non-zero octupole deformation. Also, the behaviour of the magnetic moment is shown in Fig. 6 as a two-dimensional function of $\beta_{2}$ and $\beta_{3}$ (upper-right plot) and as a function of $\beta_{3}$ (lower plot) at three different quadrupole deformations. Again, as discussed, the lower-left corner of the magnetic moment plot in Fig. 6 (upper-right) should be ignored. The plots appear to show weaker variation of $\mu$ with $\beta_{3}$ compared to the actinide cases. Here it should be 
noted that, unlike the configurations considered in the actinide nuclei, the configuration $\{\nu 5 / 2[642] \otimes \nu 3 / 2[521]\}$ is characterized by a parallel orientation of the intrinsic spins of the two orbitals, so that the intrinsic-spin contributions to the magnetic moment do not cancel. The octupole dependence of the neutron orbitals near the Fermi level of ${ }^{154} \mathrm{Nd}$ is shown in Fig. 7 for $\beta_{2}=0.3$. It is seen that both orbitals cross each other near $\beta_{3}=0.08$, consistent with the calculated 2qp energy minimum. The non-zero octupole minimum in the 2qp energy for the $K^{\pi}=4^{-}$isomeric state in ${ }^{154} \mathrm{Nd}$ might be associated with its position in the isotopic chain close to the recognized "octupole collective" nucleus ${ }^{150} \mathrm{Nd}$.

There is a notable series of $K^{\pi}=5^{-}$isomeric states observed in the nuclei ${ }^{156} \mathrm{Nd}$ and ${ }^{156,158,160} \mathrm{Sm}[18,19]$. All these states are considered to originate from the two-quasineutron configuration $\{\nu 5 / 2[642] \otimes \nu 5 / 2[523]\}$. In the present work, only the nuclei ${ }^{156} \mathrm{Nd}$ and ${ }^{160} \mathrm{Sm}$ are considered numerically, since it is expected that the results for ${ }^{156,158} \mathrm{Sm}$ will be similar. In fact, the results obtained for ${ }^{156} \mathrm{Nd}$ and ${ }^{160} \mathrm{Sm}$ are also very similar despite the difference in the $\mathrm{Z}$ number and the two units difference in N. This is seen in Figs. 8 and 9, where the behaviour of the 2qp energy and the magnetic moment is illustrated for both nuclei respectively. From the two-dimensional energy plots (upper-left parts of Figs. 8 and 9) it is seen that in both nuclei the minimum is obtained near $\beta_{2}=0.28-0.29$ and zero octupole deformation. (The blank areas in the energy plots corresponds to deformations for which the quantum numbers $5 / 2[642]$ do not appear in any leading component of the s.p. wave function due to the presence of strong parity mixing. For these deformations the configuration cannot be formed.) These results suggest that the $K^{\pi}=5^{-}$isomers in this series of nuclei are perhaps not related, or only weakly related, to the octupole degree of freedom. This conclusion is consistent with the observed relatively flat behaviour of the magnetic moment as a function of $\beta_{3}$ illustrated in the lower plots in Figs. 8 and 9. The $\beta_{3}$ dependence of the neutron orbitals near the Fermi level of ${ }^{156} \mathrm{Nd}$ is shown in Fig. 10 for $\beta_{2}=0.295$, corresponding to the 2qp energy minimum, and the "macroscopic-microscopic" value of $\beta_{2}=0.279$ with $\beta_{4}=0.098$ [23]. It is seen that although for $\beta_{2}=0.295$ and $\beta_{3}=0$ the orbitals $5 / 2[642]$ and $5 / 2[523]$ are very close to each other, they rapidly diverge with increasing $\beta_{3}$. For the "macroscopic-microscopic" deformations the divergence is even stronger.

In the nuclei ${ }^{154} \mathrm{Gd}$ and ${ }^{156} \mathrm{Gd}$, the observed isomeric state has $K^{\pi}=7^{-}$. The Nilsson diagrams for the neutron systems of these nuclei suggest two two-quasineutron configurations as possible candidates for the formation of the $7^{-}$isomer $[20,21]$. These are $\{\nu 3 / 2[651] \otimes \nu 11 / 2[505]\}$ and $\{\nu 3 / 2[402] \otimes \nu 11 / 2[505]\}$. Both of them have been tested in the present approach. The calculations show that the quantum numbers $3 / 2[651]$ disappear from the leading component of the s.p. wave function in broad ranges of the $\left(\beta_{2}, \beta_{3}\right)$ plane. On the other hand, the leading component with the quantum numbers $3 / 2[402]$ behaves more stably, though at several limited places in the $\left(\beta_{2}, \beta_{3}\right)$ plane they appear in more than one orbital (see below). Thus, although in Ref. [20] the configuration $\{\nu 3 / 2[651] \otimes \nu 11 / 2[505]\}$ is favoured, the present analysis strongly suggests that the configuration $\{\nu 3 / 2[402] \otimes \nu 11 / 2[505]\}$ is appropriate. Results obtained for both nuclei with the latter configuration are shown in Figs. 11 and 12. The upper-left plots show that in the two nuclei minima in the 2qp energy are obtained at non-zero octupole deformations. In 
${ }^{154} \mathrm{Gd}$ this is the region near $\beta_{2}=0.32$ (quite close to the experimental value 0.312 [24]) and $\beta_{3}=0.15-0.18$. The discontinuity starting at about $\beta_{2}=0.33$ and crossing the upper plots in Fig. 11 is a result of the appearance of a second orbital with the same quantum numbers $3 / 2[402]$ as the leading s.p. wave-function component, which perturbs the 2 qp energy of the configuration. This narrow region of the plot should be discounted. Nevertheless, the indication of the non-zero $\beta_{3}$ minimum in the $2 \mathrm{qp}$ energy of ${ }^{154} \mathrm{Gd}$ is clear. A similar result is observed in Fig. 12 for ${ }^{156} \mathrm{Gd}$. Here the energy minimum is placed in the region $\beta_{2}=0.34-0.36$ (again close to the experimental value $0.339[24]$ ) and $\beta_{3}=0.15-0.20$. In both nuclei the magnetic moment shows sensitivity to the octupole deformation although it is not as pronounced as in the actinide nuclei. The $\beta_{3}$ dependence of the neutron orbitals near the Fermi level of ${ }^{154} \mathrm{Gd}$ and ${ }^{156} \mathrm{Gd}$ is shown in Fig. 13 for the respective experimental values of $\beta_{2}$. It is seen that in both nuclei the 3/2[402] and $11 / 2[505]$ orbitals approach each other with increasing octupole deformation. It should be noted that these results are consistent with the previously recognized octupole collective properties of the nucleus ${ }^{154} \mathrm{Gd}$. Also, the present study suggests that these properties might extend to the neighboring isotope ${ }^{156} \mathrm{Gd}$.

\section{Conclusion}

After theoretically evaluating magnetic dipole moments for a range of $2 \mathrm{qp}$ states in eveneven actinide and rare-earth nuclei, including their dependence on quadrupole and octupole deformations, it is clear that in some cases there is great sensitivity to the octupole degree of freedom, though this is not uniformly true. In general, the greatest sensitivity to $\beta_{3}$ is found for 2-quasineutron states with anti-parallel intrinsic-spin couplings. This accounts for a large fraction of the presently know 2qp isomers in the regions considered.

It can be concluded that in the sensitive cases it would be especially useful to make accurate magnetic moment measurements, and to determine if these do indeed place useful constraints on the octupole deformation.

\section{Acknowledgements}

We acknowledge support from the UK Royal Society, STFC and AWE plc, and from the Bulgarian National Science Fund (contract DID-02/16-17.12.2009). 


\section{References}

[1] P. M. Walker and G. D. Dracoulis, Nature 399, 35 (1999).

[2] P. M. Walker, D. Ward, O. Häusser, H. R. Andrews and T. Faestermann, Nucl. Phys. A 349, 1 (1980).

[3] T. L. Shaw, V. R. Green, C.J. Ashworth, J. Rikovska, N. J. Stone, P. M. Walker and I. S. Grant, Phys. Rev. C 36, 413 (1987).

[4] J. Billowes, Nucl. Phys. A 752, 309c (2005).

[5] P. M. Walker and N. Minkov, Phys. Lett. B 694, 119 (2010).

[6] P. A. Butler and W. Nazarewicz, Rev. Mod. Phys. 68, 349 (1996).

[7] V. V. Pashkevich and V. M. Strutinsky, Sov. J. Nucl. Phys. 9, 39 (1969).

[8] J. Damgaard, H. C. Pauli, V. V. Pashkevich and V. M. Strutinsky, Nucl. Phys. A 135, 432 (1969).

[9] M. Brack, J. Damgaard, A. S. Jensen, H. C. Pauli, V. M. Strutinsky and C. Y. Wong, Rev. Mod. Phys. 44, 320 (1972).

[10] S. Cwiok, J. Dudek, W. Nazarevicz, J. Skalski and T. Werner, Comp. Phys. Comm. 46, 379 (1987).

[11] N. Minkov, S. Drenska, M. Strecker and W. Scheid, J. Phys. G: Nucl. Part. Phys. 37, 025103 (2010).

[12] S. G. Nilsson and I. Ragnarsson, Shapes and Shells in Nuclear Structure (Cambridge: Cambridge University Press, 1995).

[13] P. Ring and P. Shuck, The Nuclear Many-Body Problem (Heidelberg: Springer, 1980).

[14] H. F. Brinckmann, D. D. Clark, N. J. S. Hansen and J. Pedersen, Phys. Lett. B 5, 386 (1973).

[15] B. Bengtson, J. Jensen, M. Moszyński and H. L. Nielsen, Nucl. Phys. A 159, 249 (1970).

[16] S. E. Vandenbosch and P. Day, Nucl. Phys. 30, 177 (1962).

[17] P. G. Hansen, K. Wilsky, C. V. K. Baba and S. E. Vandenbosch, Nucl. Phys. 45, 410 (1963).

[18] G. S. Simpson, W. Urban, J. Genevey, R. Orlandi, J. A. Pinston, A. Scherillo, A. G. Smith, J. F. Smith, I. Ahmad and J. P. Greene, Phys. Rev. C 80, 024304 (2009).

[19] C. Gautherin et al., Eur. Phys. J. A 1, 391 (1998). 
[20] L. L. Riedinger, D. C. Sousa, E. G. Funk and J. W. Mihelich, Phys. Rev. C 4, 1352 (1971).

[21] J. Konijn, F. W. N. de Boer, A. van Poelgeest, W. H. A. Hesselink, M. J. A. de Voigt, H. Verheul and O. Scholten, Nucl. Phys. A 352, 191 (1981).

[22] C. J. Gallagher, Phys. Rev. 126, 1525 (1962).

[23] P. Möller, R. J. Nix, W. D. Myers and W. J. Swiatecki, At. Data Nucl. Data Tables 59, 185 (1995).

[24] S. Raman, C. W. Nestor, Jr., and P. Tikkanen, At. Data Nucl. Data Tables 78, 1 (2001).

[25] N. Minkov, P. Yotov, S. Drenska, W. Scheid, D. Bonatsos, D. Lenis and D. Petrellis, Phys. Rev. C 73, 044315 (2006).

[26] P. T. Greenlees et al., Phys. Rev. C 78, 021303(R) (2008). 

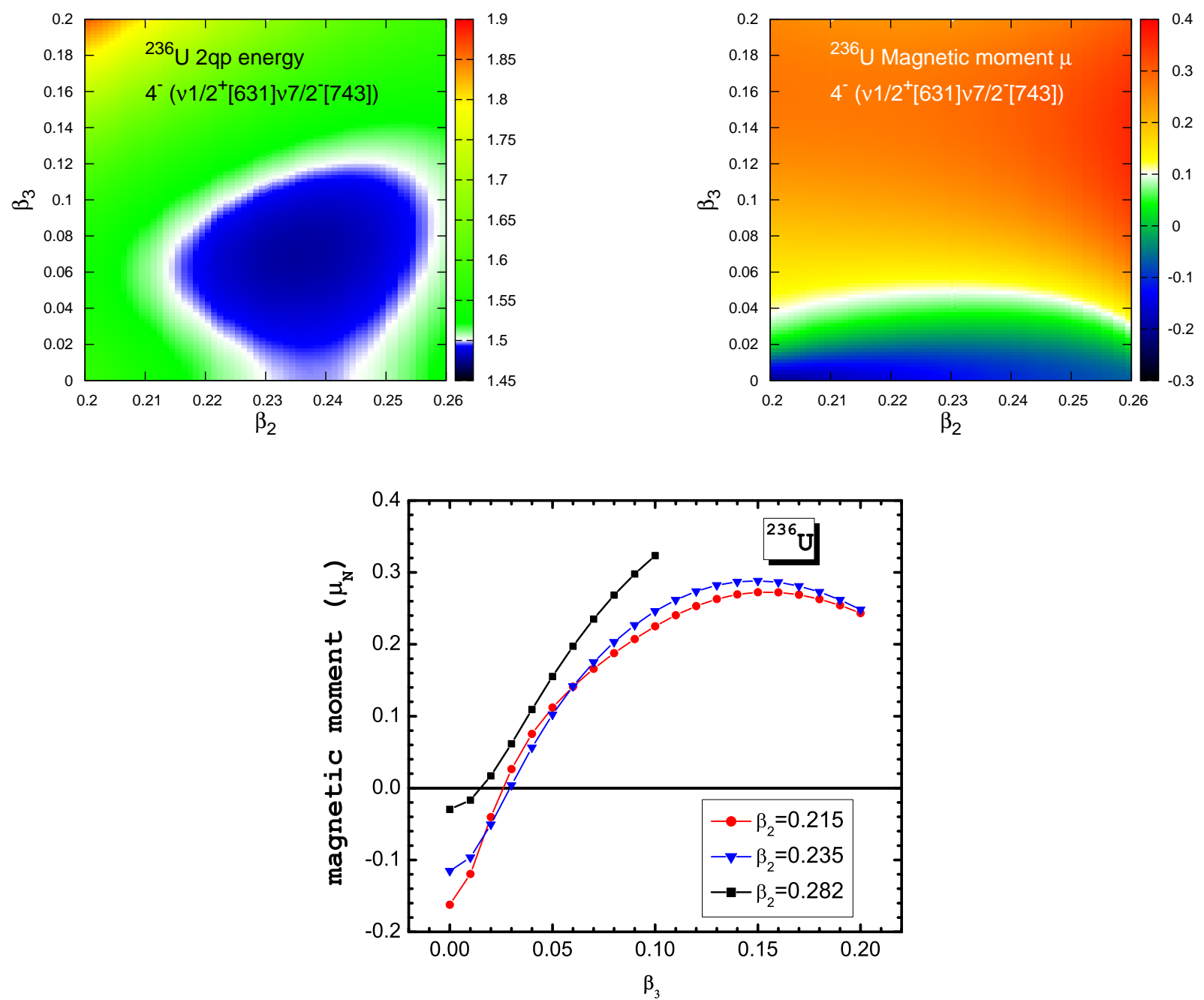

Figure 1: Calculated two-quasiparticle energy and magnetic moment of the $K^{\pi}=4^{-}$, $\{\nu 1 / 2[631] \otimes \nu 7 / 2[743]\}$ configuration in ${ }^{236} \mathrm{U}$ as a function of $\beta_{2}$ and $\beta_{3}$. 

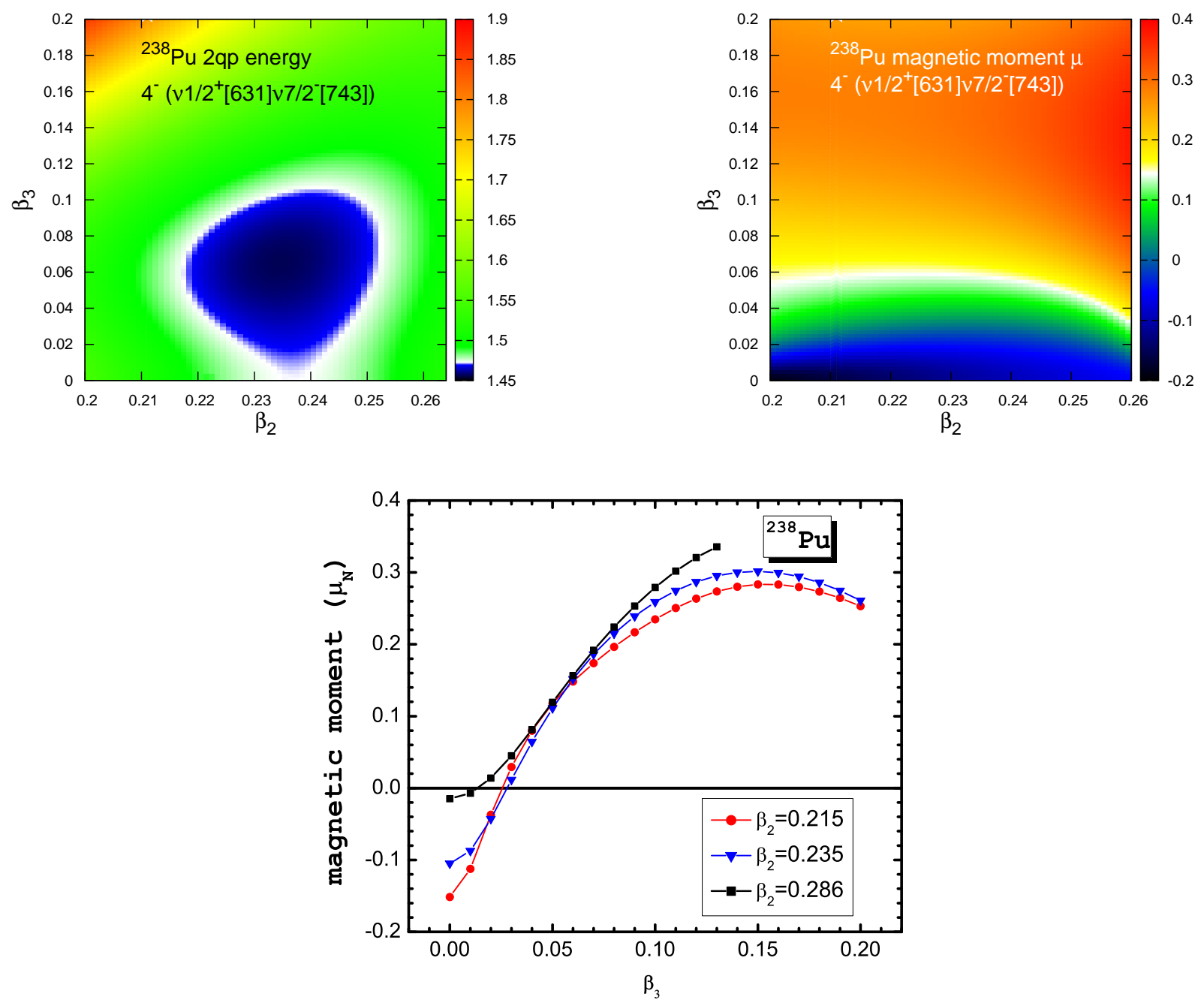

Figure 2: Calculated two-quasiparticle energy and magnetic moment of the $K^{\pi}=4^{-}$, $\{\nu 1 / 2[631] \otimes \nu 7 / 2[743]\}$ configuration in ${ }^{238} \mathrm{Pu}$ as a function of $\beta_{2}$ and $\beta_{3}$. 

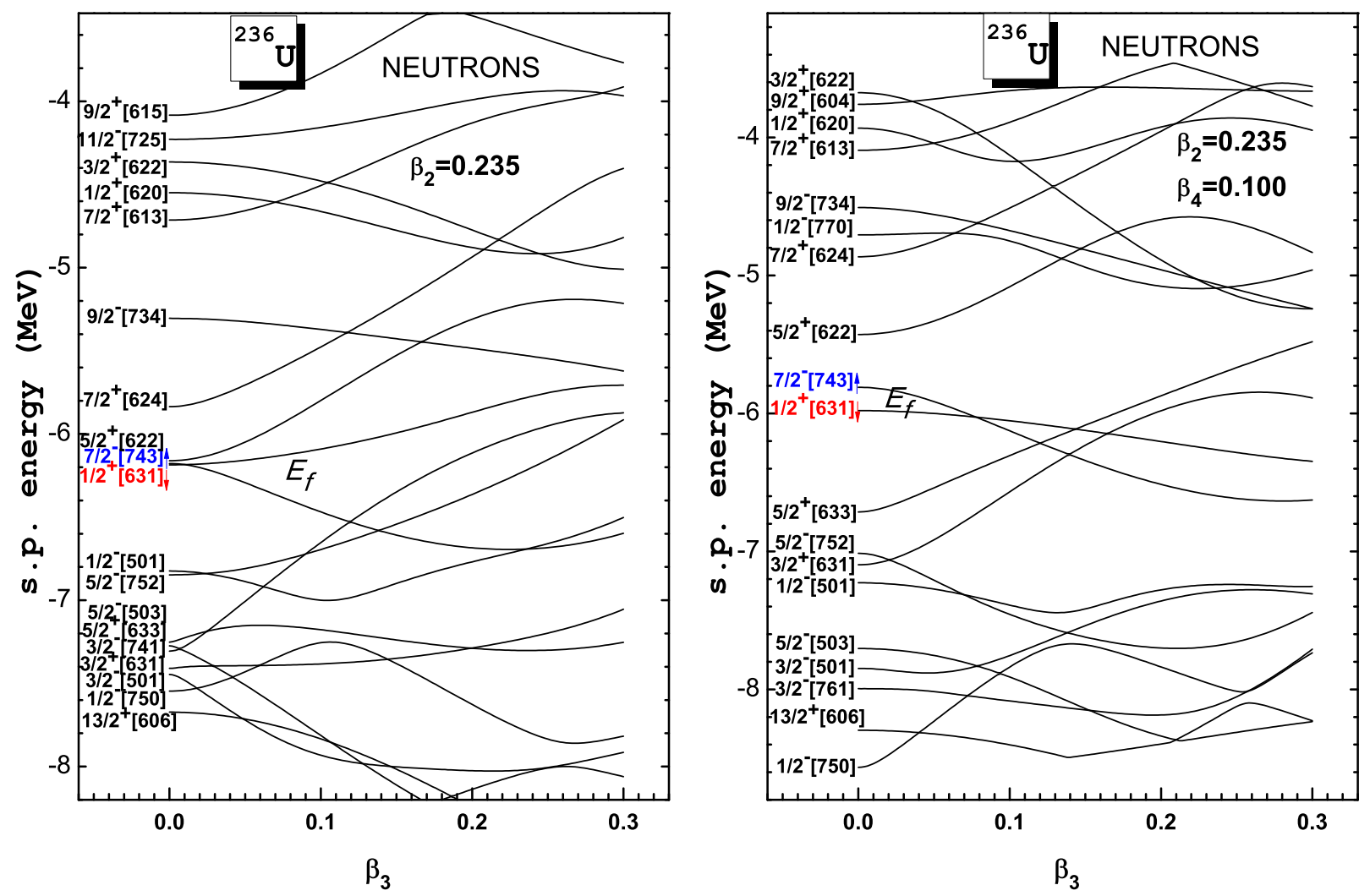

Figure 3: Neutron s.p. levels near the Fermi level $E_{f}$ of ${ }^{236} \mathrm{U}$ as functions of the octupole deformation $\beta_{3}$, with $\beta_{2}=0.235$ (left) and with the same $\beta_{2}$ and $\beta_{4}=0.100$ (right). 

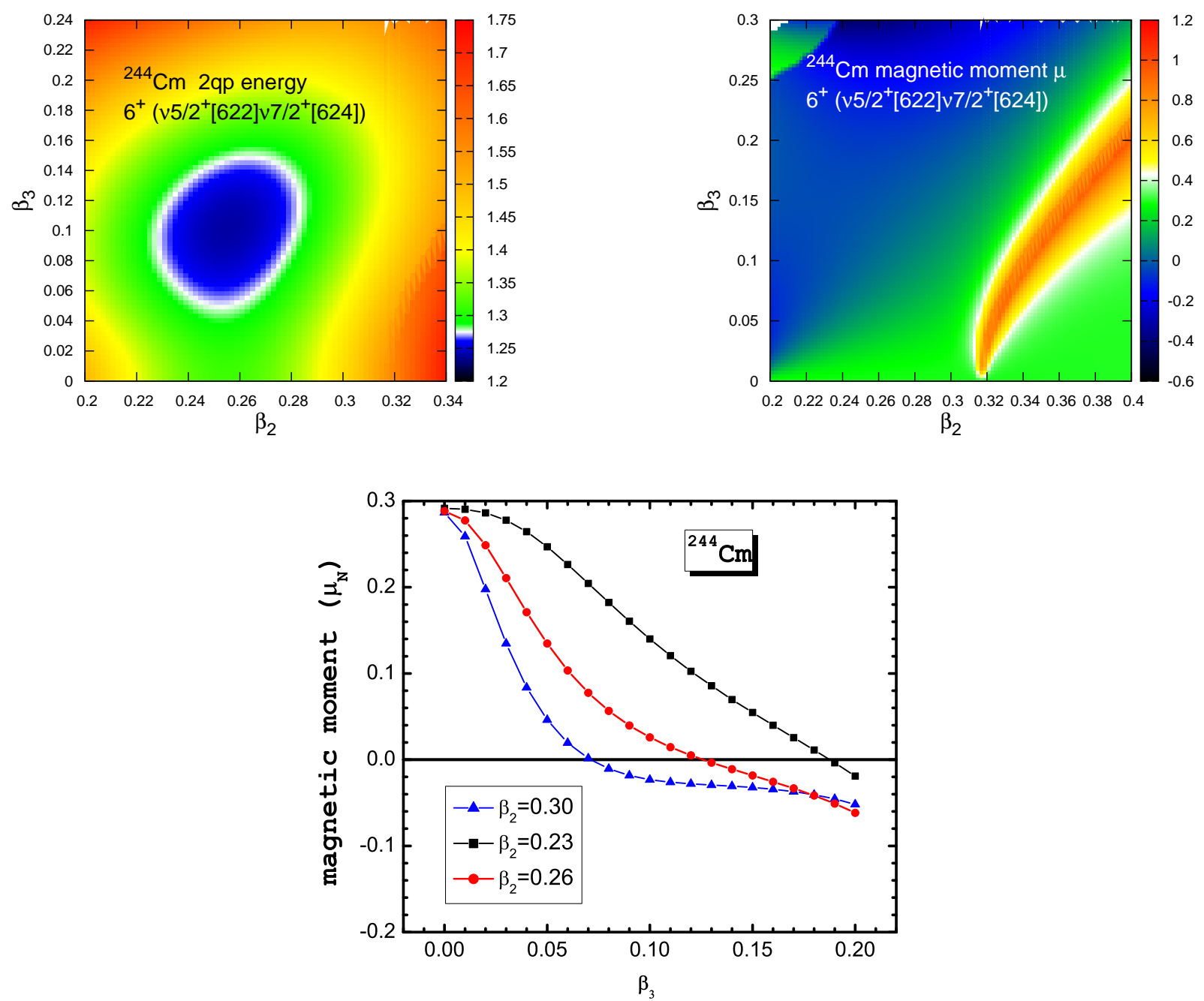

Figure 4: Calculated two-quasiparticle energy and magnetic moment of the $K^{\pi}=6^{+}$, $\{\nu 5 / 2[622] \otimes \nu 7 / 2[624]\}$ configuration in ${ }^{244} \mathrm{Cm}$ as a function of $\beta_{2}$ and $\beta_{3}$. 

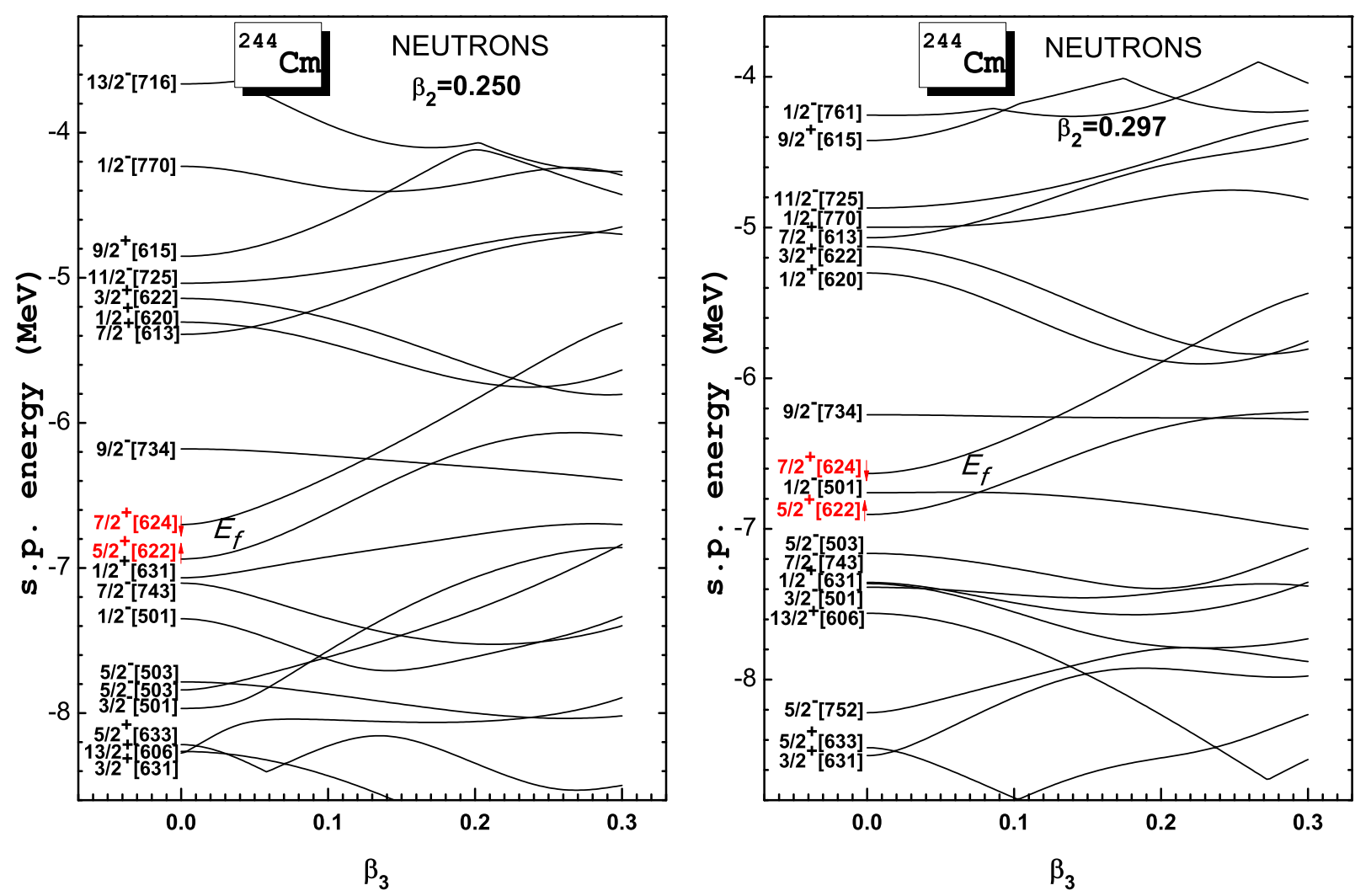

Figure 5: Neutron s.p. levels near the Fermi level $E_{f}$ of ${ }^{244} \mathrm{Cm}$ as functions of the octupole deformation $\beta_{3}$, with $\beta_{2}=0.250$ (left) and with $\beta_{2}=0.297$ (right). 

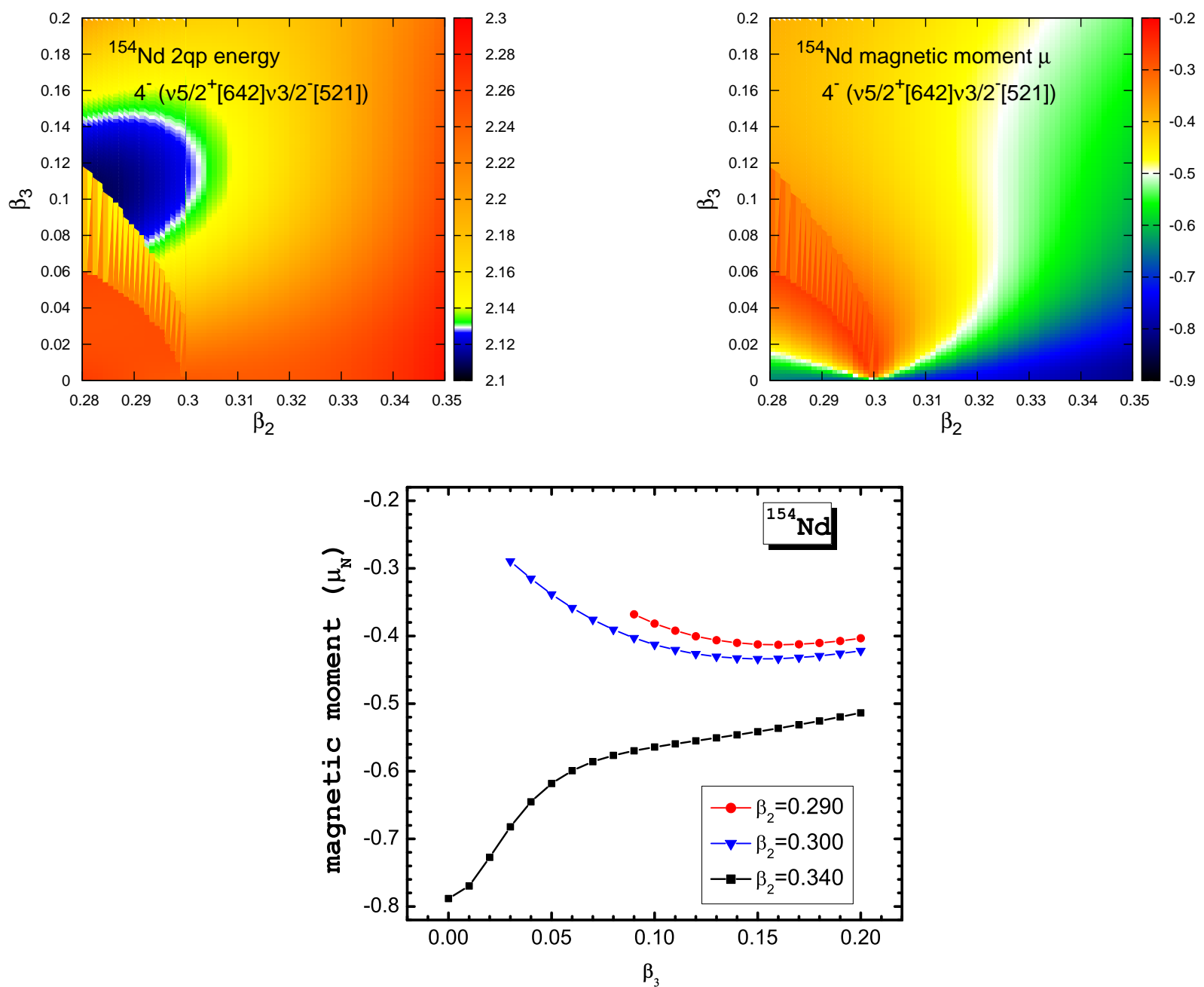

Figure 6: Calculated two-quasiparticle energy and magnetic moment of the $K^{\pi}=4^{-}$, $\{\nu 5 / 2[642] \otimes \nu 3 / 2[521]\}$ configuration in ${ }^{154} \mathrm{Nd}$ as a function of $\beta_{2}$ and $\beta_{3}$. 


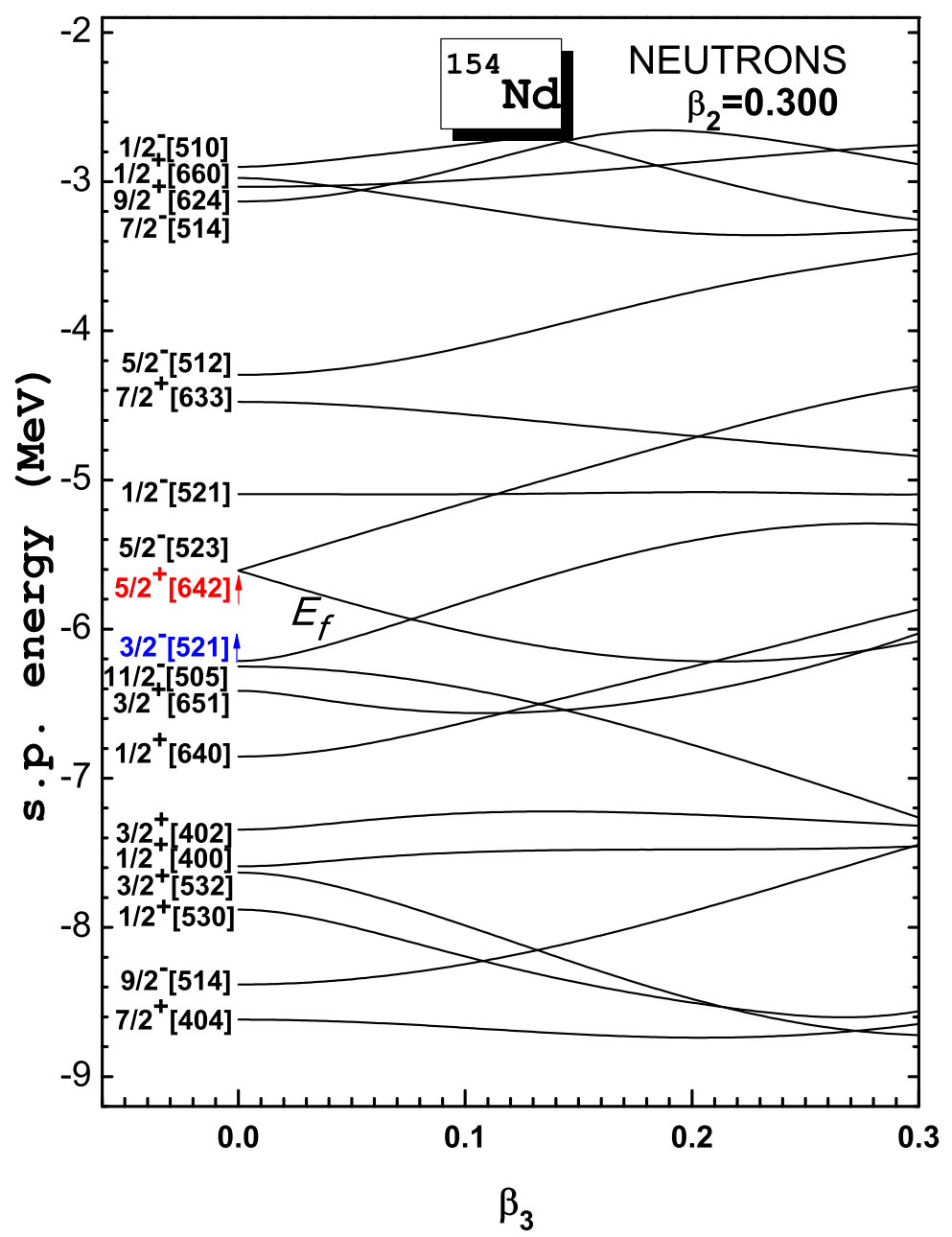

Figure 7: Neutron s.p. levels near the Fermi level $E_{f}$ of ${ }^{154} \mathrm{Nd}$ as functions of the octupole deformation $\beta_{3}$, with $\beta_{2}=0.3$. 

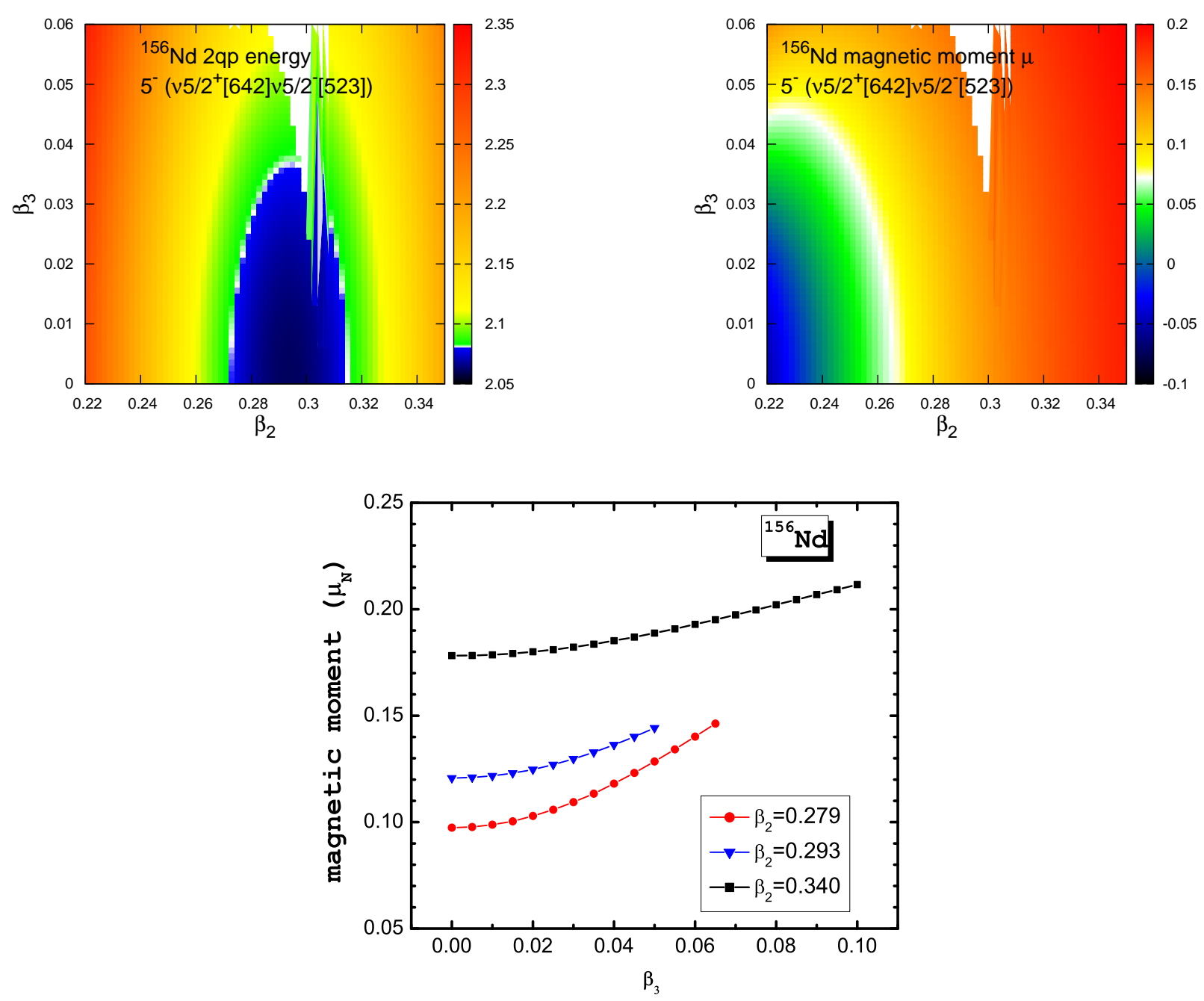

Figure 8: Calculated two-quasiparticle energy and magnetic moment of the $K^{\pi}=5^{-}$, $\{\nu 5 / 2[642] \otimes \nu 5 / 2[523]\}$ configuration in ${ }^{156} \mathrm{Nd}$ as a function of $\beta_{2}$ and $\beta_{3}$. 

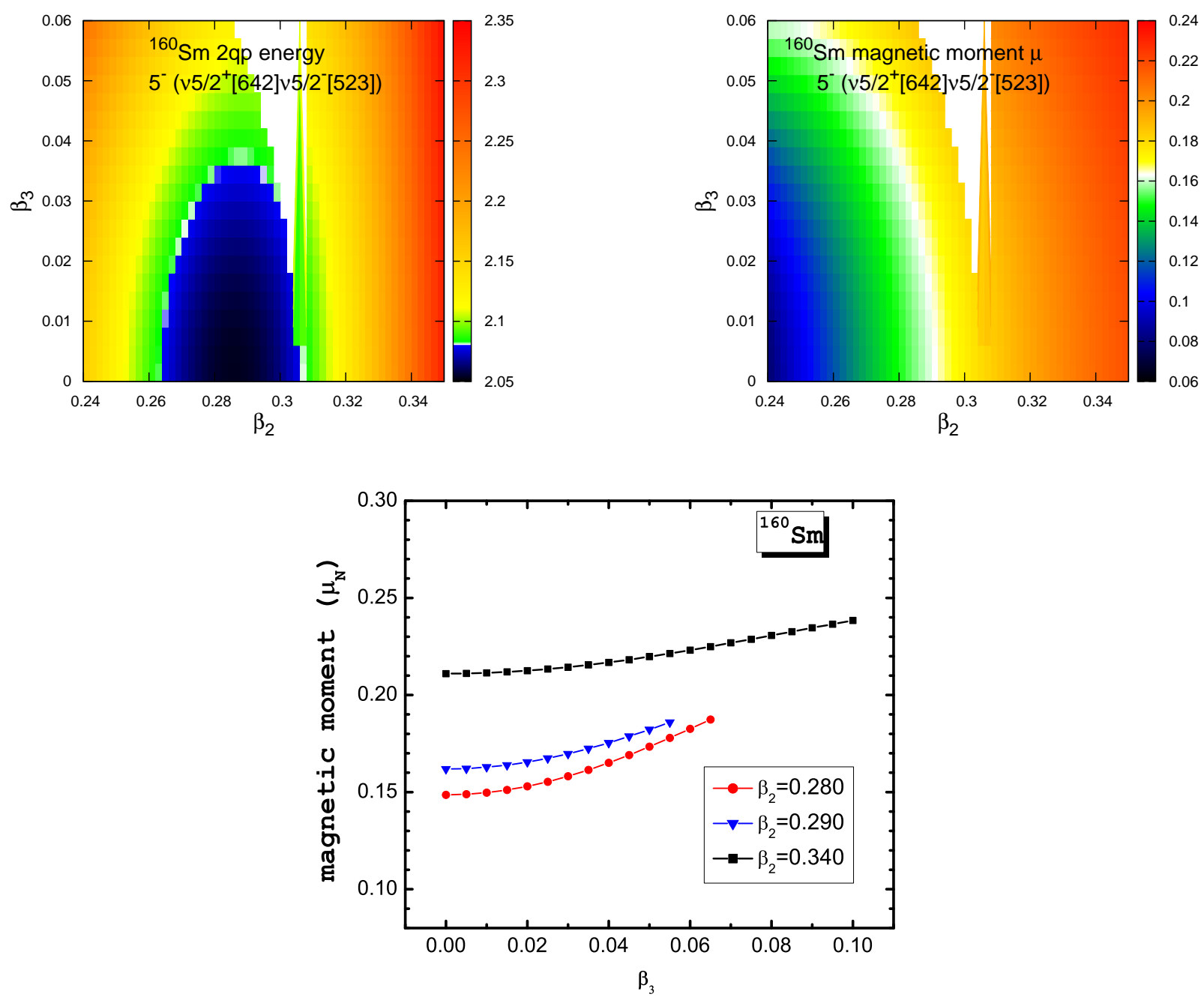

Figure 9: Calculated two-quasiparticle energy and magnetic moment of the $K^{\pi}=5^{-}$, $\{\nu 5 / 2[642] \otimes \nu 5 / 2[523]\}$ configuration in ${ }^{160} \mathrm{Sm}$ as a function of $\beta_{2}$ and $\beta_{3}$. 

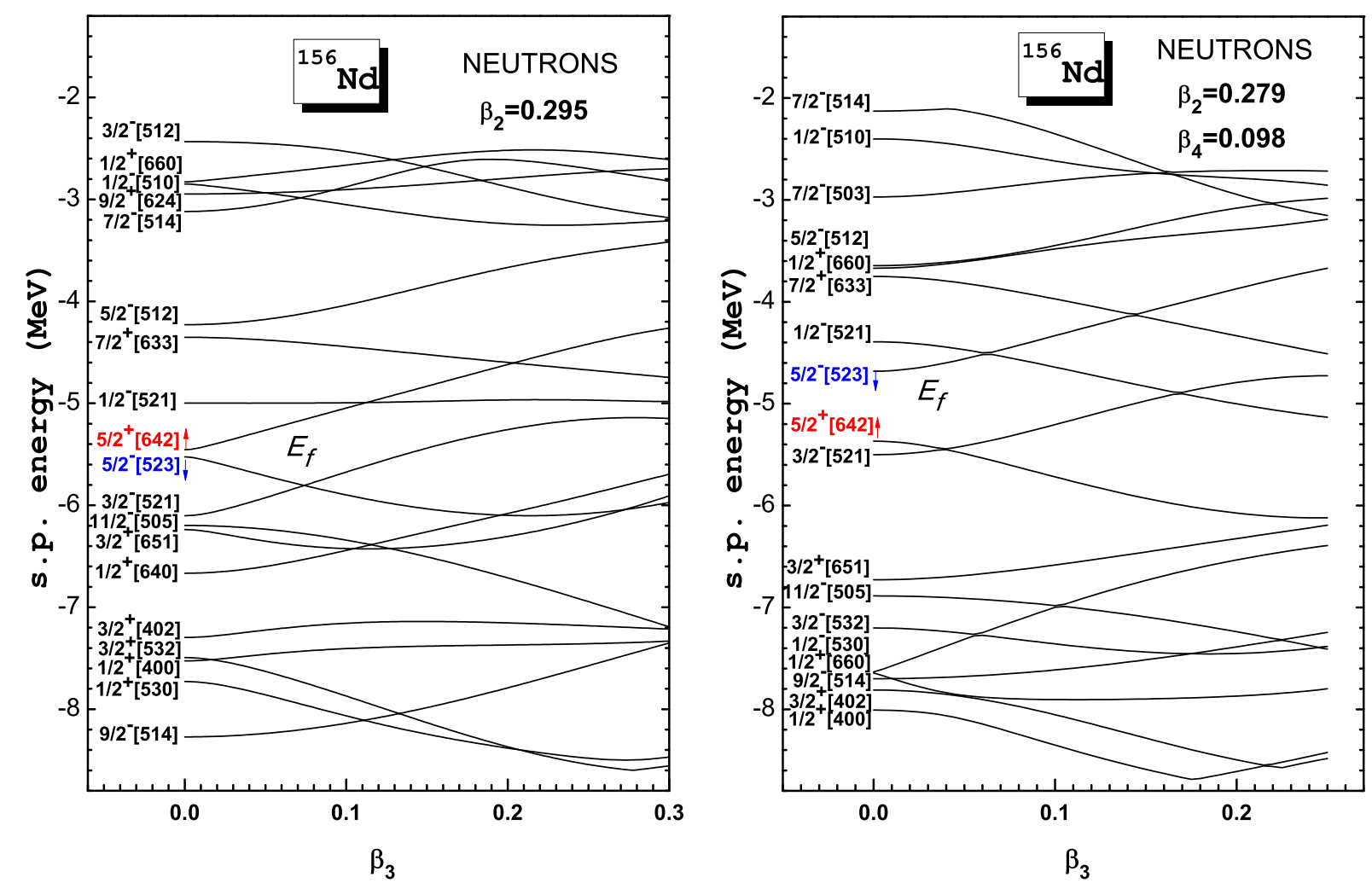

Figure 10: Neutron s.p. levels near the Fermi level $E_{f}$ of ${ }^{156} \mathrm{Nd}$ as functions of the octupole deformation $\beta_{3}$, with $\beta_{2}=0.295$ (left) and with $\beta_{2}=0.279$ and $\beta_{4}=0.098$ (right). 

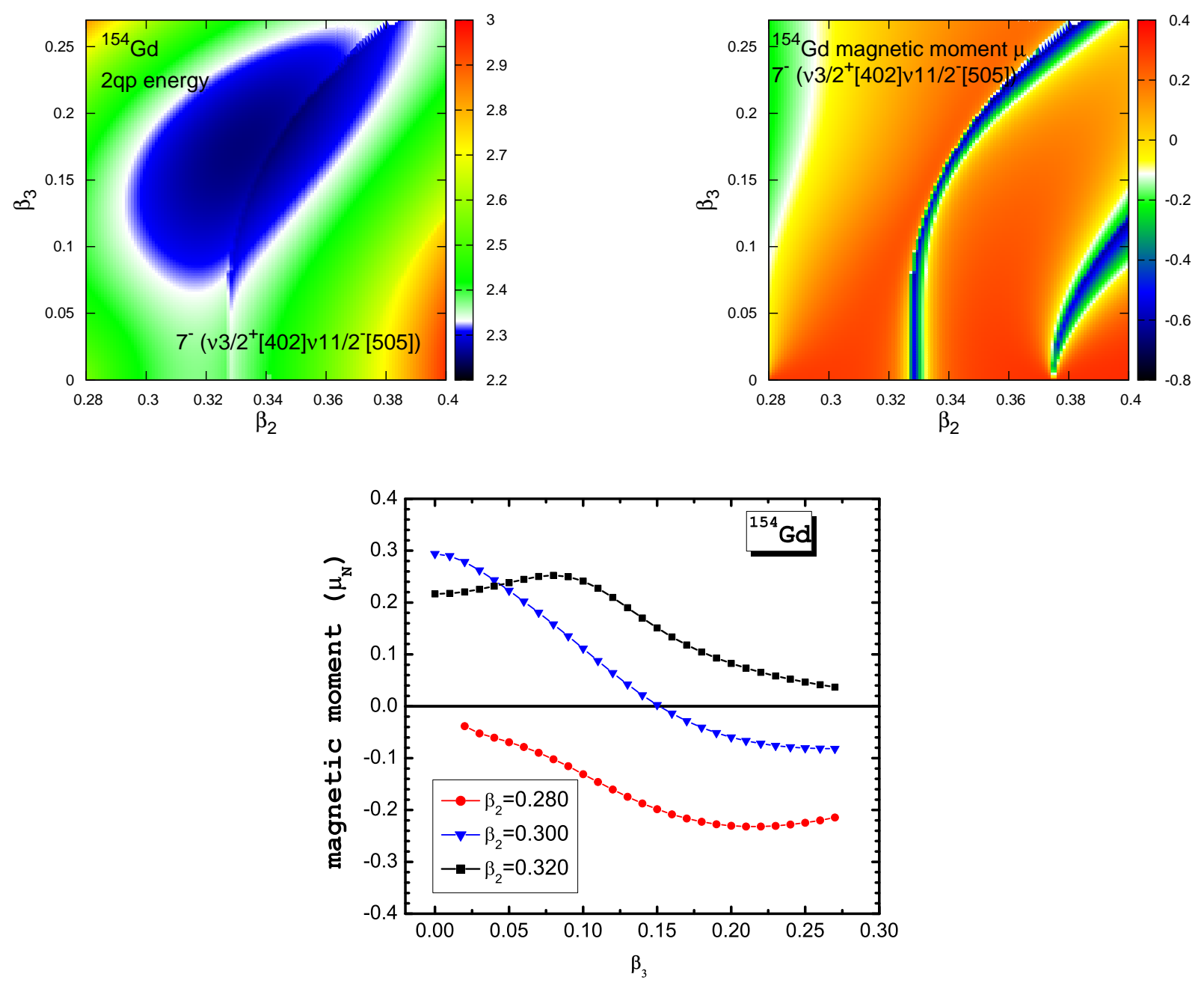

Figure 11: Calculated two-quasiparticle energy and magnetic moment of the $K^{\pi}=7^{-}$, $\{\nu 3 / 2[402] \otimes \nu 11 / 2[505]\}$ configuration in ${ }^{154} \mathrm{Gd}$ as a function of $\beta_{2}$ and $\beta_{3}$. 

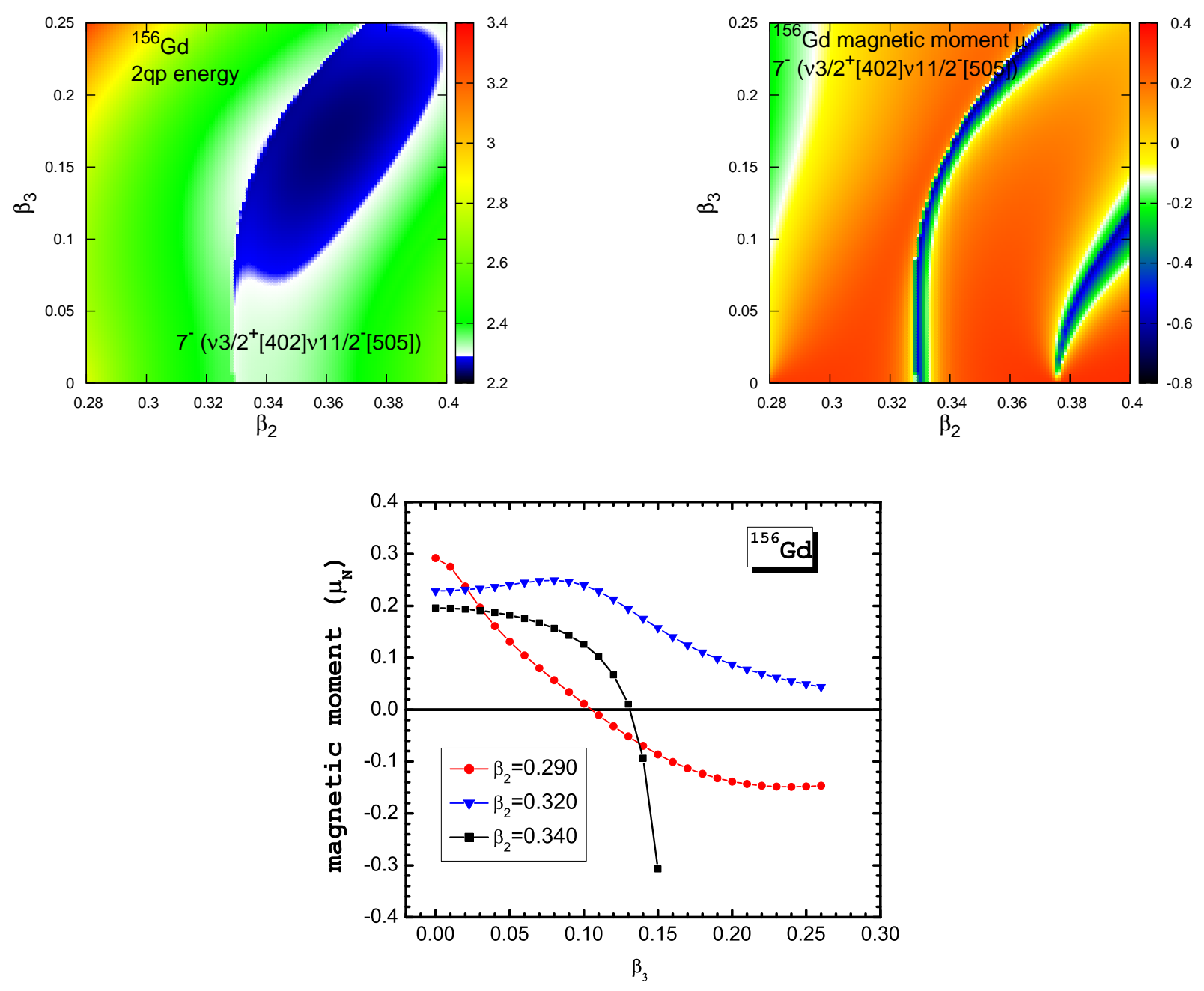

Figure 12: Calculated two-quasiparticle energy and magnetic moment of the $K^{\pi}=7^{-}$, $\{\nu 3 / 2[402] \otimes \nu 11 / 2[505]\}$ configuration in ${ }^{156} \mathrm{Gd}$ as a function of $\beta_{2}$ and $\beta_{3}$. 

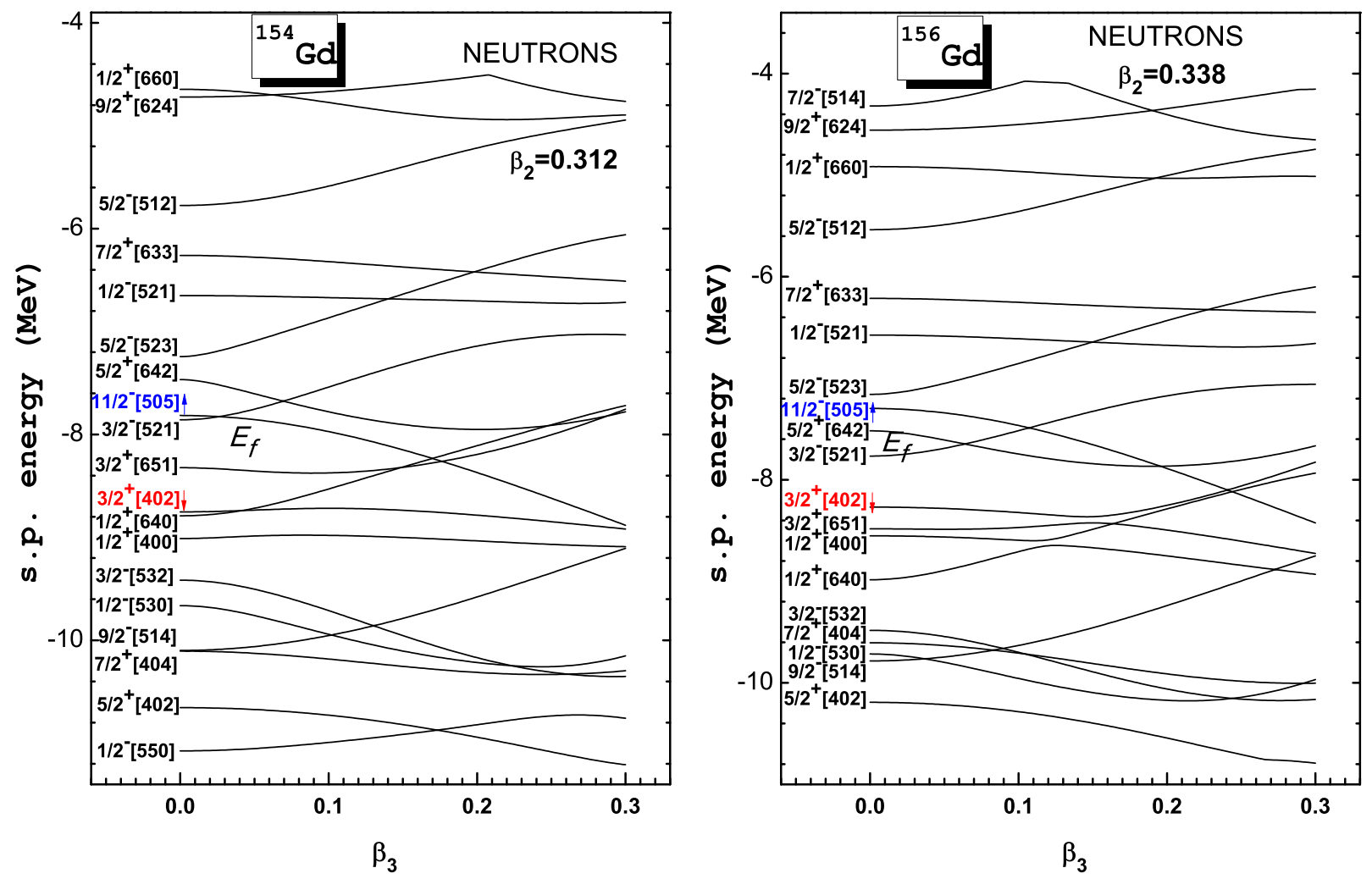

Figure 13: Neutron s.p. levels near the Fermi level $E_{f}$ as functions of the octupole deformation $\beta_{3}$ for ${ }^{154} \mathrm{Gd}$ with $\beta_{2}=0.312$ (left) and for ${ }^{156} \mathrm{Gd}$ with $\beta_{2}=0.338$ (right). 